\title{
Quasi-semidefinite eigenvalue problem and applications
}

\author{
L. Grubišić and J. Tambača \\ Department of Mathematics, Faculty of Science, University of Zagreb, \\ Bijenička 30, 10000 Zagreb, Croatia \\ luka.grubisic@math.hr, josip.tambaca@math.hr
}

PACS 02.30.Tb,02.70.-c, 02.10.Ud

DOI $10.17586 / 2220-8054-2017-8-2-180-187$

\begin{abstract}
In this note, we study the eigenvalue problem for a class of block operator matrix pairs. Our study is motivated by an analysis of abstract differential algebraic equations. Such problems frequently appear in the study of complex systems, e.g. differential equations posed on metric graphs, in mixed variational formulation.
\end{abstract}

Keywords: block operator matrices, metric graphs, spectral theory and eigenvalue problems.

Received: 15 January 2017

Revised: 2 February 2017

\section{Introduction}

In this note, we will consider mixed variational formulations of evolution problems as abstract differential algebraic equations. More generally, abstract differential algebraic equations can be seen as a good tool to study the evolution of any system with constraints. Their theory and numerical analysis is fairly well known in the finite dimensional case, see eg. [1], but the corresponding results for infinite dimensional abstract differential algebraic equations are at an early stage of development, see $[2,3]$.

Let us note that simulations of complex systems, where one considers several physical regimes simultaneously can be described by a state vector in an appropriate product space called the state space. Experience indicates that mixed variational formulations are easier to combine in a state space formulation. In the mixed approach, the "agreement" between models/regimes is enforced a constraint in the state space.

Our approach to mixed variational problems is through the theory of $2 \times 2$ block operator matrices [4] and the representation theorems for quasi-definite quadratic forms by such operators from $[5,6]$.

Formally, we start from the problem of finding $u \in L^{2}(0, T, X)$ and $p \in L^{2}(0, T, Y), T>0$ so that:

$$
\left[\begin{array}{cc}
M & 0 \\
0 & 0
\end{array}\right] \frac{d}{d t}\left[\begin{array}{l}
u \\
p
\end{array}\right]+\left[\begin{array}{cc}
K & B \\
D^{*} & C
\end{array}\right]\left[\begin{array}{l}
u \\
p
\end{array}\right]=\left[\begin{array}{l}
f_{1} \\
f_{2}
\end{array}\right]
$$

holds in the state space $X \otimes Y$. As in [2,3,7], the time derivative is to be interpreted in the distributional sense and $X$ and $Y$ are assumed to be Hilbert spaces. We will call $X \otimes Y$ the state space. It is a Hilbert space with usual product space structure and we will write:

$$
\psi=x \oplus y=\left[\begin{array}{l}
x \\
y
\end{array}\right] \in X \otimes Y
$$

for its elements, the state vectors.

An important class of model problems which can formally be posed in the form (1) originates from problems in fluid mechanics modeled by Stokes, Oseen or Navier-Stokes equations linearized about nonzero velocity, see [3] for some details. In this note, we will be interested in the homogeneous problem associated to the problem (1):

$$
\left[\begin{array}{cc}
M & 0 \\
0 & 0
\end{array}\right] \frac{d}{d t}\left[\begin{array}{l}
u \\
p
\end{array}\right]+\left[\begin{array}{cc}
K & B \\
D^{*} & C
\end{array}\right]\left[\begin{array}{l}
u \\
p
\end{array}\right]=0
$$

and its fundamental solutions $\psi(t)=\exp (\lambda t) \psi_{0}$. From the Floquet theorem, we know that $\lambda \in \mathbb{C}$ and $\psi_{0} \in X \otimes Y$ must satisfy the algebraic problem:

$$
\lambda\left[\begin{array}{cc}
M & 0 \\
0 & 0
\end{array}\right] \psi_{0}+\left[\begin{array}{cc}
K & B \\
D^{*} & C
\end{array}\right] \psi_{0}=0 .
$$


Note that eigenvalue problem (3) also covers the case of some special second order systems. For instance, in the absence of damping we have:

$$
\left[\begin{array}{cc}
M & 0 \\
0 & 0
\end{array}\right] \frac{d^{2}}{d t^{2}}\left[\begin{array}{l}
u \\
p
\end{array}\right]+\left[\begin{array}{cc}
K & B \\
D^{*} & C
\end{array}\right]\left[\begin{array}{l}
u \\
p
\end{array}\right]=0
$$

Equation (4) leads to the consideration of a quadratic eigenvalue problem. It is a special type of the quadratic eigenvalue problem which can be transformed into a linear eigenvalue problem by a simple change of spectral variable.

We will restrict our analysis to considering equations (4) where operator $M$ is a bounded positive semi-definite operator and the block operator matrix is quasi-semidefinite. For the purposes of this note, a block operator matrix is called positive quasi semi-definite if it defines a boundedly invertible self-adjoint operator and has the structure:

$$
\mathcal{K}=\left[\begin{array}{ll}
K & B \\
B^{*} & 0
\end{array}\right]
$$

where $B$ is the closed range operator and $K$ is positive semidefinite operator in the sense of Kato, [8]. To tackle both problems in a unified manner, we will analyze the generalized resolvents:

$$
\mathcal{R}_{1}: z \mapsto\left[\begin{array}{cc}
K-z M & B \\
B^{*} & 0
\end{array}\right]^{-1}, \quad \mathcal{R}_{2}: z \mapsto\left[\begin{array}{cc}
K-z^{2} M & B \\
B^{*} & 0
\end{array}\right]^{-1}
$$

as $2 \times 2$ block operator matrices. The only assumption which we will make is that there are complex numbers $z_{1}, z_{2} \in \mathbb{C}$ such that $\mathcal{R}_{1}\left(z_{1}\right)$ and $\mathcal{R}_{2}\left(z_{2}\right)$ are bounded and that in addition, $K^{-1}$ is compact. The terminology for quasi-definite block operator matrices comes from [9] and the references therein.

\section{Matrix analysis of the generalized resolvent}

In this section, we will present matrix analysis of the generalized resolvents (5) under the additional assumption that $X$ and $Y$ are finite dimensional. The computations are motivated by the considerations from [10,11]. We will restrict our analysis to the following setting. Without reducing the level of generality, we consider a general Hermitian (self-adjoint) block $2 \times 2$ matrix of the form:

$$
\mathcal{K}=\left[\begin{array}{cc}
K & B \\
B^{*} & 0
\end{array}\right]
$$

where matrix $B$ is such that $\left\|\left(B^{*} B\right)^{-1}\right\|<\infty$. Such block matrices were called quasi-semidefinite in [9]. In this case, there exists a unitary matrix $Q=\left[\begin{array}{ll}Q_{1} & Q_{2}\end{array}\right]$ and a lower triangular matrix $R$ which is invertible and:

$$
B=\left[\begin{array}{ll}
Q_{1} & Q_{2}
\end{array}\right]\left[\begin{array}{c}
R^{*} \\
0
\end{array}\right] \text {. }
$$

This directly implies the following for the range space of the operator $B$ and the null space (kernel) of the operator $B^{*}$ :

$$
\begin{aligned}
\operatorname{Im}(B) & =\operatorname{Im}\left(Q_{1}\right), \\
\operatorname{Null}\left(B^{*}\right) & =\operatorname{Im}\left(Q_{2}\right) .
\end{aligned}
$$

We define the unitary block matrix:

$$
\mathbb{Q}=\left[\begin{array}{ll}
Q & 0 \\
0 & I
\end{array}\right]=Q \oplus I
$$

In the state space formulation, we will henceforth use the notation $A \oplus B$ to denote the block diagonal matrix with diagonal blocks $A$ and $B$. With these definitions, we compute:

$$
\mathbb{Q}^{*} \mathcal{K} \mathbb{Q}=\left(\left[\begin{array}{ll}
Q_{1} & Q_{2}
\end{array}\right] \oplus I\right)^{*} \mathcal{K}\left(\left[\begin{array}{ll}
Q_{1} & Q_{2}
\end{array}\right] \oplus I\right)=\left[\begin{array}{ccc}
K_{11} & K_{12} & R^{*} \\
K_{21} & K_{22} & 0 \\
R & 0 & 0
\end{array}\right] .
$$

The analysis of [12] implies the following technical lemma. 
Lemma 1. Assume that $B$ is such that $\left\|\left(B^{*} B\right)^{-1}\right\|<\infty$ and $Q_{1}, Q_{2}$ and $R$ are as in (6). Then,

$$
\mathcal{K}=\left[\begin{array}{cc}
K & B \\
B^{*} & 0
\end{array}\right]
$$

is invertible if and only if $Q_{2}^{*} K Q_{2}=K_{22}$ is invertible. In that case $u$ and $p$ which satisfy:

$$
\left[\begin{array}{ll}
K & B \\
B^{*} & 0
\end{array}\right]\left[\begin{array}{l}
u \\
p
\end{array}\right]=\left[\begin{array}{l}
f \\
g
\end{array}\right]
$$

are given by:

$$
\begin{aligned}
& u=\left[\begin{array}{c}
Q_{1} R^{-1} g \\
Q_{2} K_{22}^{-1}\left(Q_{2}^{*} f-K_{21} R^{-1} g\right)
\end{array}\right], \\
& p=Q_{1}^{*} f-K_{11} R^{-1} g-K_{12} K_{22}^{-1}\left(Q_{1}^{*} f-K_{21} R^{-1} g\right) .
\end{aligned}
$$

Proof. According to [9], we have:

$$
\operatorname{Null}(\mathcal{K})=\left(\operatorname{Null}(K) \cap \operatorname{Null}\left(B^{*}\right)\right) \otimes \operatorname{Null}(B)
$$

for the null space of $\mathcal{K}$. The assumptions on the matrix $B$ imply that $\operatorname{Null}(B)=\{0\}$ and $\operatorname{Null}\left(B^{*}\right)=\operatorname{Im}\left(Q_{2}\right)$, and so, if $K_{22}=Q_{2}^{*} K Q_{2}$ is invertible, so is $\left(\operatorname{Null}(K) \cap \operatorname{Null}\left(B^{*}\right)\right)$ trivial. This proves the first claim. To finish the proof of the theorem, note that (6) implies:

$$
\left[\begin{array}{ccc}
K_{11} & K_{12} & R \\
K_{21} & K_{22} & 0 \\
R^{*} & 0 & 0
\end{array}\right]\left[\begin{array}{c}
Q_{1}^{*} u \\
Q_{2}^{*} u \\
p
\end{array}\right]=\left[\begin{array}{c}
Q_{1}^{*} f \\
Q_{2}^{*} f \\
g
\end{array}\right]
$$

and the claim follows by equating the matrix identity block element by block element.

Lemma 1 directly allows for the block matrix analysis of the resolvents and was used in [12] to analyze the eigenvalue problem associated to the resolvent $\mathcal{R}_{1}$. In particular it holds:

$$
\mathbb{Q}^{*} \mathcal{R}_{i}(z) \mathbb{Q}\left[\begin{array}{c}
f_{1} \\
f_{2} \\
g
\end{array}\right]=\left[\begin{array}{c}
R^{-1} g \\
\mathcal{R}_{22, i}(z)\left(f_{2}-\left(K_{21}-z^{i} M_{21}\right) R^{-1} g\right) \\
-\left(K_{12}-z^{i} M_{12}\right) \mathcal{R}_{22, i}(z)\left(f_{2}-\left(K_{21}-z^{i} M_{21}\right) R_{1}^{-1} g\right)+f_{1}-\left(K_{11}-z^{i} M_{11}\right) R^{-1} g
\end{array}\right],
$$

where

$$
\mathcal{R}_{22, i}(z):=\left(K_{22}-z^{i} M_{22}\right)^{-1} .
$$

Here, we see that the singularities of the functions $z \mapsto \mathcal{R}_{i}(z)$ and $z \mapsto \mathcal{R}_{22, i}(z)$ coincide. Let $\rho_{i}\left(K_{22}, M_{22}\right)=$ $\left\{z \in \mathbb{C}:\left\|\left(K_{22}-z^{i} M_{22}\right)^{-1}\right\|<\infty\right\}$ be the resolvent set of $\mathcal{R}_{22, i}$. Its complement $\operatorname{Spec}_{i}\left(K_{22}, M_{22}\right)=$ $\mathbb{C} \backslash \rho_{i}\left(K_{22}, M_{22}\right)$ is called the spectrum of $\mathcal{R}_{i}$. In particular, when $K_{22}$ is a positive definite Hermitian (selfadjoint) matrix, then for each $\lambda \in \operatorname{Spec}_{i}\left(K_{22}, M_{22}\right)$ there is a vicinity $\mathcal{U} \subset \rho_{i}\left(K_{22}, M_{22}\right)$ such that the expansion:

$$
\mathcal{R}_{i}(z)=\frac{1}{z-\lambda} P_{i}+\mathcal{H}_{i}(z), \quad z \in \mathcal{U},
$$

holds. Here, $\mathcal{H}_{i}$ is a holomorphic operator valued function and $\operatorname{Im}(P)=\operatorname{Null}\left(K_{22}-\lambda^{i} M_{22}\right)$. This result follows from the celebrated Keldysh' theorem, see $[13,14]$. Let us note that in the case in which we assume that $K_{22}$ is positive definite, as will be prototypical for the applications which we have in mind, then we will study the resolvents associated with the operator $K_{22}^{-1} M_{22}$ and so obtain the results for the resolvents $\mathcal{R}_{i}, i=1,2$, see [15] and the references therein. Note that [15] is obtained in the Hilbert space setting and this will allow a direct generalization in the next section.

\section{The main results}

In this section, we concentrate on the operator equivalent of the matrix results. Let us assume that $X$ and $Y$ are Hilbert spaces. Then, we assume we have operators $K: X \rightarrow X$ and $B: Y \rightarrow X$ such that:

(A1): $K$ is self-adjoint and positive semi-definite;

(A2): $B$ is closed;

(A3): $\left\|B^{*}(I+K)^{-1 / 2}\right\|<\infty$;

(A4): $Y=\operatorname{Im}\left(B^{*}(I+K)^{1 / 2}\right)$;

(A5): The restriction of the operator $K$ on the space $\operatorname{Im}\left(B^{*}\right)$ is positive definite (in the sense of quadratic forms, see below). 
The restriction of the operator $K$ on the space $\operatorname{Null}\left(B^{*}\right) \subset X$, denoted by $K_{22}$, is defined as the operator representation, in the sense of Kato [8], of the quadratic form:

$$
k_{22}(u, v):=\left(K^{1 / 2} u, K^{1 / 2} v\right), \quad u, v \in \operatorname{Null}\left(B^{*}\right) \cap \operatorname{Dom}\left(K^{1 / 2}\right) .
$$

Here, $K^{1 / 2}$ is a self-adjoint operator defined by spectral calculus and satisfies the requirements of the Kato's second representation theorem and $V:=\operatorname{Dom}\left(K^{1 / 2}\right) \subset X$ denotes the operator domain of $K^{1 / 2}$. The operator $K_{22}$ is now a self adjoint and positive definite operator from $\operatorname{Null}\left(B^{*}\right)$ to $\operatorname{Null}\left(B^{*}\right)$ such that:

$$
\left(K_{22}^{1 / 2} u, K_{22}^{1 / 2} v\right)=k_{22}(u, v), \quad u, v \in \operatorname{Null}\left(B^{*}\right) \cap \operatorname{Dom}\left((I+K)^{1 / 2}\right)
$$

and $\operatorname{Dom}\left(K_{22}^{1 / 2}\right)=\operatorname{Null}\left(B^{*}\right) \cap \operatorname{Dom}\left((I+K)^{1 / 2}\right.$.

The null space of the closed operator is closed and the assumptions (A1) and (A2) together with the closed range theorem imply:

$$
X=\operatorname{Im}(B) \oplus \operatorname{Null}\left(B^{*}\right) .
$$

Based on this decomposition, which is an abstract version of the Hodge-Helmholtz decomposition for the Stokes operator, we will seek a block $3 \times 3$ representation of the resolvents $\mathcal{R}_{1}$ and $\mathcal{R}_{2}$ in the state space:

$$
X \otimes Y=\left(\operatorname{Im}(B) \oplus \operatorname{Null}\left(B^{*}\right)\right) \otimes Y=\operatorname{Im}(B) \otimes \operatorname{Null}\left(B^{*}\right) \otimes Y,
$$

where we have tacitly identified ismorphic Hilbert spaces. Let us now consider the following forms:

$$
\begin{aligned}
k_{11}(u, v) & =\left(K^{1 / 2} u, K^{1 / 2} v\right), & & u, v \in \operatorname{Im}(B) \cap\left(\operatorname{Dom}(I+K)^{1 / 2}\right), \\
k_{21}(u, v) & =\left(K^{1 / 2} u, K^{1 / 2} v\right), & & u \in \operatorname{Im}(B) \cap\left(\operatorname{Dom}(I+K)^{1 / 2}\right), v \in \operatorname{Null}\left(B^{*}\right) \cap\left(\operatorname{Dom}(I+K)^{1 / 2}\right), \\
b(u, v) & =\left(u, B^{*} v\right), & & u \in Y, v \in V \subset X .
\end{aligned}
$$

Further, let $K_{11}$ be the operator representation of $k_{11}$ in the sense of Kato, and let $R$ be the maximal operator such that:

$$
(u, R v)=b(u, v), \quad u \in Y, v \in \operatorname{Im}(B) \cap \operatorname{Dom}\left((I+K)^{1 / 2}\right) .
$$

Then, by the assumptions (A3) and (A4), the operator $R: \operatorname{Im}(B) \rightarrow Y$ is closed and has a bounded inverse.

Note that the forms $k_{12}$ and $k_{21}$ need not have operator representations. Also, they are conjugate to each other as forms since $K$ is self-adjoint. However, the operator $K_{R}$ defined by:

$$
\left(K_{R} u, v\right)=k_{21}\left(R^{-1} u, K_{22}^{-1 / 2} v\right), \quad u \in Y, v \in \operatorname{Null}\left(B^{*}\right),
$$

is a bounded operator. Assumptions (A1) and (A3) also imply that $R^{-*} K_{11} R$ is a bounded self-adjoint operator.

We now present the main theorem on the block operator representation of the resolvents $\mathcal{R}_{i}, i=1,2$.

Theorem 1. Let $M$ be a bounded and self-adjoint semi-definite operator on $\operatorname{Im}(B) \otimes \operatorname{Null}\left(B^{*}\right)$ and let $K$ and $B$ be operators which satisfy (A1)-(A5). Let further $K_{11}$ and $K_{22}$ be the operators as defined in (7)-(9) and let $K^{-1}$ be compact. Then for $z \in \mathbb{C}$ the operator $\mathcal{R}_{i}(z)$ is bounded if and only if $\mathcal{R}_{22, i}(z)=\left(K_{22}-z^{i} M_{22}\right)^{-1}$ is bounded. Here we assumed the obvious notation for the block $2 \times 2$ representation of a bounded operator $M$ on $\operatorname{Im}(B) \otimes \operatorname{Null}\left(B^{*}\right)$.

Proof. Recall from $[5,6,9]$ that the operator:

$$
\mathcal{K}=\left[\begin{array}{ll}
K & B \\
B^{*} & 0
\end{array}\right]
$$

is semibounded from below and so due to its quasi semi-definitnes we can justify the following computation in the product space $\left(\operatorname{Im}(B) \times \operatorname{Null}\left(B^{*}\right)\right) \otimes Y$ :

$$
\begin{aligned}
& \mathcal{R}_{i}(z)= \\
& \left(R^{-*} \oplus K_{22}^{-1 / 2} \oplus I\right)\left[\begin{array}{ccc}
R^{-*} K_{11} R^{-1}-z^{i} R^{-*} M_{11} R^{-1} & K_{R}-z^{i} R^{-*} M_{12} K_{22}^{-1 / 2} & I \\
K_{R}^{*}-z^{i} K_{22}^{-1 / 2} M_{21} R^{-1} & I-z^{i} K_{22}^{-1 / 2} M_{22} K_{22}^{-1 / 2} & 0 \\
I & 0 & 0
\end{array}\right]^{-1} \quad\left(R^{-1} \oplus K_{22}^{-1 / 2} \oplus I\right) .
\end{aligned}
$$

The $3 \times 3$ block operator matrix in the middle has only bounded entries and it is, using the same technique as in Lemma 1, boundedly invertible if and only if:

$$
\left\|\left(I-z^{i} K_{22}^{-1 / 2} M_{22} K_{22}^{-1 / 2}\right)^{-1}\right\|<\infty .
$$

Since $K_{22}$ has a compact inverse and $M_{22}$ is bounded this is equivalent, see [15], to $\left\|\left(K_{22}-z^{i} M_{22}\right)^{-1}\right\|<\infty$ which was the claim of the theorem. 
Recall that under the assumption that $K_{22}^{-1}$ is compact and $K_{22}$ is positive definite and self-adjoint operator we can again apply Keldysch's theorem on the operator function $z \mapsto\left(K_{22}-z^{i} M_{22}\right)^{-1}$. For any $i=1,2$ and $\lambda_{i} \in \rho_{i}\left(K_{22}, M_{22}\right)$ there exists a vicinity $\mathcal{U}_{i} \subset \rho_{i}\left(K_{22}, M_{22}\right)$ and an operator $P_{i}$ and a holomorphic operator valued function $\mathcal{H}_{i}$ such that:

$$
\mathcal{R}_{22, i}(z)=\left(K_{22}-z^{i} M_{22}\right)^{-1}=\frac{1}{z-\lambda_{i}} P_{i}+\mathcal{H}_{i}(z), \quad z \in \mathcal{U}_{i}
$$

Let us assume (A1)-(A5) and in addition let $K^{-1}$ be compact then

$$
\mathcal{K}=\left[\begin{array}{ll}
K & B \\
B^{*} & 0
\end{array}\right]
$$

is invertible. Based on the analysis in the state $\operatorname{space} \operatorname{Im}(B) \otimes \operatorname{Null}\left(B^{*}\right) \otimes Y$ and following the steps from [11,12], we can construct $2 \times 2$ block matrix representation of $\mathcal{K}^{-1} \mathcal{M}$ in the product space $\operatorname{Null}\left(B^{*}\right) \otimes(\operatorname{Im}(B) \otimes Y)$ which has the form:

$$
\mathcal{K}^{-1} \mathcal{M}=L_{1}\left[\begin{array}{ll}
A & \\
& N
\end{array}\right] L_{1}^{-1},
$$

where $A$ is a self-adjoint compact operator and $N$ is a nilpotent operator such that $N^{2}=0$ and $L_{1}$ and $L_{1}^{-1}$ are bounded operators. We see that the eigenvalues of the operator $\mathcal{K}^{-1} \mathcal{M}$ are directly mapped by to the eigenvalues of $\mathcal{R}_{i}$ by either inverting them or by inverting their squares. Note that $A$ might have a zero as an eigenvalue. In this case, we say that this zero corresponds to the eigenvalue at infinity of the original eigenvalue problem associated with $\mathcal{R}_{i}, i=1,2$. The whole invariant space of $\mathcal{K}^{-1} \mathcal{M}$ associated with the operator $N$ corresponds to the eigenvalue at infinity of $\mathcal{R}_{i}, i=1,2$. On the other hand, the eigenvalues of $\mathcal{K}^{-1} \mathcal{M}$ in the invariant subspace associated with the operator $A$ are all semisimple.

\section{A model problem}

As a model problem, we consider the curved rod model from [16, 17]. A discussion of the model is beyond the scope of this article. In the weak formulation model is given for the unknowns $y$ and $\theta$ being the displacement of the middle curve and the infinitesimal rotation of the cross-section. Having in mind the mixed formulation of the model, see [18] for more details, we set:

$$
V:=H_{0}^{1}\left(0, l ; \mathbb{R}^{3}\right) \times H_{0}^{1}\left(0, l ; \mathbb{R}^{3}\right) \subset L^{2}\left(0, l ; \mathbb{R}^{3}\right) \times L^{2}\left(0, l ; \mathbb{R}^{3}\right)=: X, \quad Y:=L^{2}\left(0, l ; \mathbb{R}^{3}\right),
$$

and define the bilinear forms:

$$
\begin{aligned}
& k: V \times V \rightarrow \mathbb{R}, \quad k((y, \theta),(\tilde{y}, \tilde{\theta}))=\int_{0}^{l} \mathbf{Q H Q}^{*} \partial_{s} \theta \cdot \partial_{s} \tilde{\theta} d s, \\
& b: Y \times V \rightarrow \mathbb{R}, \quad b(n,(\tilde{y}, \tilde{\theta}))=\int_{0}^{l} n \cdot\left(\partial_{s} \tilde{y}+\mathbf{t} \times \tilde{\theta}\right) d s,
\end{aligned}
$$

where $\mathbf{H} \in \mathbb{R}^{3 \times 3}$ is a symmetric positive definite matrix describing the elastic properties of the material the rod is

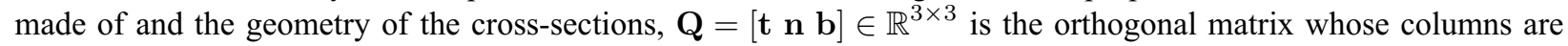
the tangent, the normal and the binormal of the of the middle line of the curved rod, see Fig. 1. The elastic energy of the rod is given in the form $k$, while the form $b$ defines the inextensibility and unshearability conditions of the $\operatorname{rod}\left(\partial_{s} y+\mathbf{t} \times \theta=0\right)$. Thus the elements of the state space $X \otimes Y$ are given by $(y, \theta) \oplus n$ and also include the Lagrange multiplier (being the contact force) related to the inextensibility and unshearability conditions.

Since $V$ is dense in $X$ and the form $k$, defined on the domain $V$, is closed and positive semidefinite in $X$ and so it defines the self-adjoint positive semi-definite operator $K$ in $X$ in the sense of Kato. Furthermore, this choice of $V$ allows us to conclude that the operator $B^{*}: X \rightarrow Y$ such that:

$$
\left(n, B^{*}(y, \theta)\right)_{Y}=b(n,(y, \theta))
$$

is a closed operator and $\left\|B^{*}(I+K)^{-1 / 2}\right\|<\infty$. Here it is central that $\operatorname{Im}(I+K)^{-1 / 2}=V$. To prove the assertion (A4) we use the inf $-\sup$ estimates for $B$. The details can be found in [18]. The form $m$, which defines 


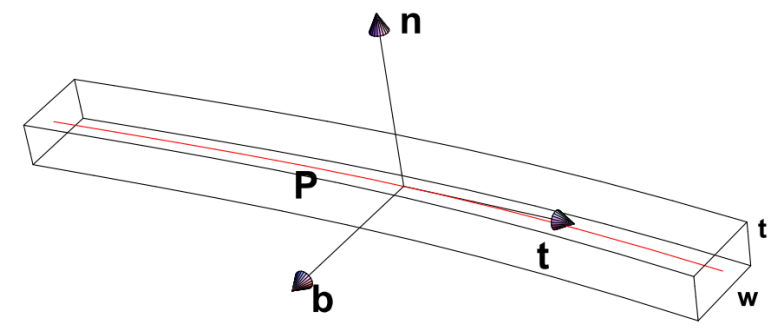

FIG. 1. A 3D elastic strut modeled by the curved rod model on the middle line of length $l$ which is parametrized by $P:[0, l] \rightarrow \mathbb{R}^{3}$

the $M$, is given by the bounded form:

$$
m: X \times X \rightarrow \mathbb{R}, \quad m((y, \theta),(\tilde{y}, \tilde{\theta}))=\int_{0}^{l} \rho A y \cdot \tilde{y} d s,
$$

and $\rho$ and $A$ are the density and the area of the cross section of the rod.

\subsection{Endovascular stents}

Following the approach from [17] it is possible to consider the curved rod equations on the metric graph $\mathcal{N}=(\mathcal{V}, \mathcal{E})$, where $\mathcal{V}$ are vertices and $\mathcal{E}$ are edges which are assumed to be parametrized by $P^{i}:\left[0, l_{i}\right] \rightarrow \mathbb{R}^{3}$, $i=1, \ldots, \# \mathcal{E}$. Here $\# \mathcal{E}<\infty$ is the number of edges in $\mathcal{N}$. As before deformation of the $i$ th edge is described by $u^{i}=\left(y^{i}, \theta^{i}\right)$, the displacement of the $i$ th middle curve and the infinitesimal rotation of its cross-section. We further impose the restriction that between edges the displacements and the rotations of the cross-sections have to be continuous. Thus, for the domain of the form of the elastic energy, we have:

$$
\begin{aligned}
V=\left\{\mathbf{u}=\left(u^{1}, \cdots, u^{\# \mathcal{E}}\right) \in \prod_{i=1}^{\# \mathcal{E}} H^{1}\left(0, l_{i} ; \mathbb{R}^{3}\right) \times H^{1}\left(0, l_{i} ; \mathbb{R}^{3}\right):\right. \\
\\
\left.u^{i}\left(\left(P^{i}\right)^{-1}(v)\right)=u^{j}\left(\left(P^{j}\right)^{-1}(v)\right), \forall v \in \mathcal{V}, v \in e^{i} \cap e^{j}, i, j=1, \ldots, \# \mathcal{E}\right\} .
\end{aligned}
$$

For the spaces $X$ and $Y$, we choose:

$$
\begin{aligned}
X & =\prod_{i=1}^{\# \mathcal{E}} L^{2}\left(0, l_{i} ; \mathbb{R}^{3}\right) \times L^{2}\left(0, l_{i} ; \mathbb{R}^{3}\right), \\
Y & =\prod_{i=1}^{\# \mathcal{E}} L^{2}\left(0, l_{i} ; \mathbb{R}^{3}\right) \times \mathbb{R}^{3} \times \mathbb{R}^{3} .
\end{aligned}
$$

The forms that define the problem are now given by:

$$
\begin{aligned}
& k: V \times V \rightarrow \mathbb{R}, \quad k(\mathbf{u}, \tilde{\mathbf{u}})=\sum_{i=1}^{\# \mathcal{E}} \int_{0}^{l_{i}} \mathbf{Q}^{i} \mathbf{H}^{i} \mathbf{Q}^{i} * \partial_{s} \theta^{i} \cdot \partial_{s} \tilde{\theta}^{i} d s \\
& b: Y \times V \rightarrow \mathbb{R}, \quad b(\mathbf{n}, \tilde{\mathbf{u}})=\sum_{i=1}^{\# \mathcal{E}} \int_{0}^{l_{i}} n^{i} \cdot\left(\partial_{s} \tilde{y}^{i}+\mathbf{t}^{i} \times \tilde{\theta}^{i}\right) d s+\alpha \cdot \sum_{i=1}^{\# \mathcal{E}} \int_{0}^{l_{i}} \tilde{y}^{i} d s+\beta \cdot \sum_{i=1}^{\# \mathcal{E}} \int_{0}^{l_{i}} \tilde{\theta}^{i} d s .
\end{aligned}
$$

Here, we used the notation:

$$
\mathbf{n}=\left(n^{1}, \ldots, n^{\# c E}, \alpha, \beta\right) \in Y
$$

for the Lagrange multipliers in the mixed formulations; $n^{i}$ is the contact force associated to the $i$ th edge while $\alpha$ and $\beta$ are multipliers associated to fixing the overall center of mass and overall infinitesimal rotation.

With this, we again obtain the resolvent:

$$
\mathcal{K}-z^{2} \mathcal{M}=\left[\begin{array}{cc}
K-z^{2} M & B \\
B^{*} & 0
\end{array}\right]
$$


where the operator $K$ is the "stiffness" operator, the operator $B$ implements the inextensibility of the middle curve of all edges and ushearability of cross-sections of all edges of the stent and fixing the overall center of mass and rotation of the whole stent and the "mass" operator $M$ is defined by the bounded form:

$$
m: X \times X \rightarrow \mathbb{R}, \quad m(\mathbf{u}, \tilde{\mathbf{u}})=\sum_{i=1}^{\# \mathcal{E}} \int_{0}^{l_{i}} \rho^{i} A^{i} y^{i} \cdot \tilde{y}^{i} d s .
$$

Since the boundary conditions for the stent problem are homogeneous Neumann type nonuniqueness of the solution of the problem is associated. Thus, by fixing the overall translation and rotation, we obtain uniqueness of the problem, i.e. the operator $K$ is invertible on Null $B^{*}$. More details on the model and its properties can be found in [18].

This construction of the operator $B$ and the space $Y$ illustrates a use of the freedom in formulating the mixed problem so that (A1)-(A5) hold. The inf - sup analysis of $B$ can be performed in the metric graph setting following a similar argument as in the single rod case to show that (A4) holds. For details see [18]. Also, as is known from the Stokes problem, the freedom in the choice of $Y$, which is there the pressure space, is directly used to satisfy condition (A4) possibly by restricting the size of $Y$. A description of the code which was used to generate Fig. 2, as well as discretization details, is based on [19].
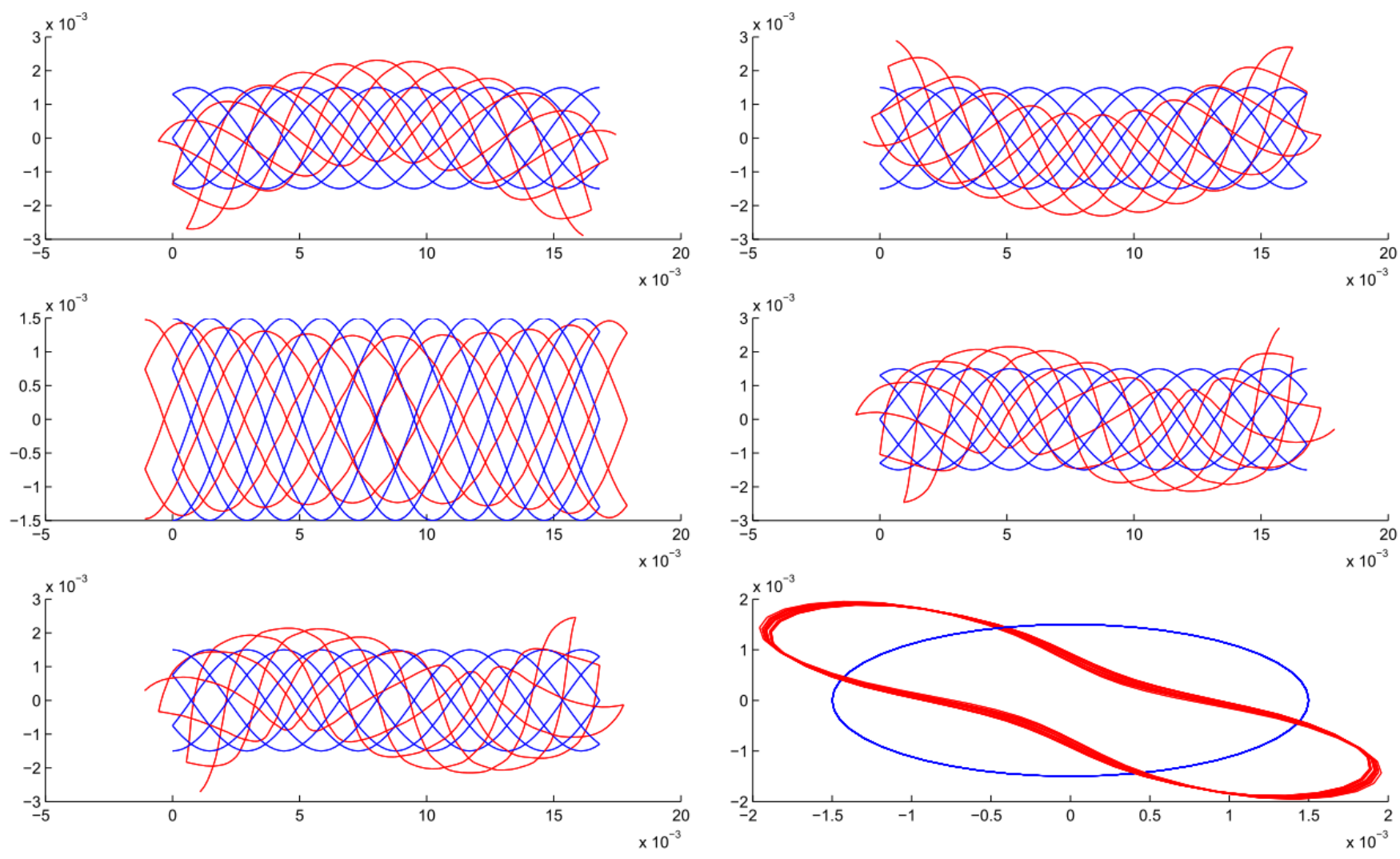

FIG. 2. A metric graph model of an endovascular stent. Six lowermost eigenmodes of the Palmaz stent computed with the MATLAB based on [19]

\section{Acknowledgements}

This research has been supported by the grant HRZZ-9345 of the Croatian Science Foundation. We gratefully acknowledge the support. We use the MATLAB [19]. 


\section{References}

[1] Kunkel P., Mehrmann V. Differential-algebraic equations. EMS Textbooks in Mathematics. European Mathematical Society (EMS), Zürich, 2006.

[2] Altmann R., Levajković T., Mena H. Operator differential-algebraic equations with noise arising in fluid dynamics. Monatshefte für Mathematik, 2016, P. 1-40.

[3] Emmrich E., Mehrmann V. Operator differential-algebraic equations arising in fluid dynamics. Comput. Methods Appl. Math., 2013, 13 (4), P. 443-470.

[4] Tretter C. Spectral theory of block operator matrices and applications. Imperial College Press, London, 2008.

[5] Grubišić L., Kostrykin V., Makarov K.A., Veselić K. Representation theorems for indefinite quadratic forms revisited. Mathematika, 2013, 59 (1), P. 169-189.

[6] Grubišić L., Kostrykin V., Makarov K.A., Veselić K. The Tan $2 \Theta$ theorem for indefinite quadratic forms. J. Spectr. Theory, 2013, 3 (1), P. 83-100.

[7] Tartar L. An introduction to Navier-Stokes equation and oceanography, 1 of Lecture Notes of the Unione Matematica Italiana. SpringerVerlag, Berlin; UMI, Bologna, 2006.

[8] Kato T. Perturbation Theory for Linear Operators. Springer-Verlag, Berlin, 1980.

[9] Veselić I., Veselić K. Spectral gap estimates for some block matrices. Oper. Matrices, 2015, 9 (2), P. 241-275.

[10] Cliffe K.A., Garratt T.J., Spence A. Eigenvalues of the discretized Navier-Stokes equation with application to the detection of Hopf bifurcations. Adv. Comput. Math., 1993, 1 (3-4), P. 337-356.

[11] Stykel T. Low-rank iterative methods for projected generalized Lyapunov equations. Electron. Trans. Numer. Anal., 2008,30, P. 187-202.

[12] Cliffe K.A., Garratt T.J., Spence A. Eigenvalues of block matrices arising from problems in fluid mechanics. SIAM J. Matrix Anal. Appl., 1994, 15 (4), P. 1310-1318

[13] Markus A.S., Macaev V.I. On the spectral theory of holomorphic operator-valued functions in Hilbert space. Funkcional. Anal. i Priložen., 1975, 9 (1), P. 76-77.

[14] Mennicken R., Möller M. Non-self-adjoint boundary eigenvalue problems. In North-Holland Mathematics Studies, 192, North-Holland Publishing Co., Amsterdam, 2003.

[15] Nakić I. On the correspondence between spectra of the operator pencil $A-\lambda B$ and of the operator $B^{-1} A$. Glas. Mat. Ser. III, 2016, 51 (71), P. 197-221.

[16] Jurak M., Tambača J. Linear curved rod model. General curve. Math. Models Methods Appl. Sci., 2001 , 11 (7), P. 1237-1252.

[17] Čanić S., Tambača J. Cardiovascular stents as PDE nets: 1D vs. 3D. IMA J. Appl. Math., 2012 , 77 (6), P. $748-770$.

[18] Grubišić L., Iveković J., Tambača J., Žugec B. Mixed formulation of the equilibrium problem for elastic stents. Submitted to Rad HAZU, 2017, URL: http://arxiv.org/abs/1703.05074.

[19] Iveković J. Numerical method for the model of linearized stents. Diploma thesis, Department of Mathematics, Faculty of Science, University of Zagreb, 2015. 